\title{
Süßgetränke als Dickmacher
}

\section{Eine randomisierte Studie an holländischen Grundschulkindern macht süße Erfrischungsgetränke als wichtige Ursache für die verbreitete Adipositas bei Kindern und Jugend- lichen aus.}

— Die Zunahme der Adipositas bei Kindern und Jugendlichen ist mittlerweile ein Phänomen, das man bereits bei einem flüchtigen Blick über den Pausenhof feststellen kann. Kein Klassenfoto, auf dem nicht auf den ersten Blick mindestens fünf Kinder mit starkem Übergewicht auszumachen sind. Im Vergleich zur Zeit vor 20 und 30 Jahren hat sich hier etwas fundamental verändert, wobei die Gründe für diese Entwicklung vielfältig und in ihrer Gewichtung auch noch nicht geklärt sind.

Eine über 18 Monate laufende randomisierte und kontrollierte Studie an 641 Schulkindern in Holland im Alter zwischen vier Jahren und zehn Monaten und elf Jahren und elf Monaten bringt nun etwas Licht ins Dunkel. Jeweils die Hälfte der Kinder erhielt für jeden Tag der Woche je eine $250 \mathrm{ml}$-Dose mit einem Getränk, das entweder mit zuckerfreiem Süßstoff oder mit $26 \mathrm{~g}$ Glukose gesüßt war. Die Flüssigkeit der identisch aussehenden Dosen schmeckte ähnlich süß, der Kaloriengehalt betrug allerdings, je nach Inhalt, 0 bzw. $104 \mathrm{kcal}$.

Zum vorgesehenen Studienende nach 18 Monaten hatten 26\% der Kinder den Gebrauch der Studiengetränke ein-

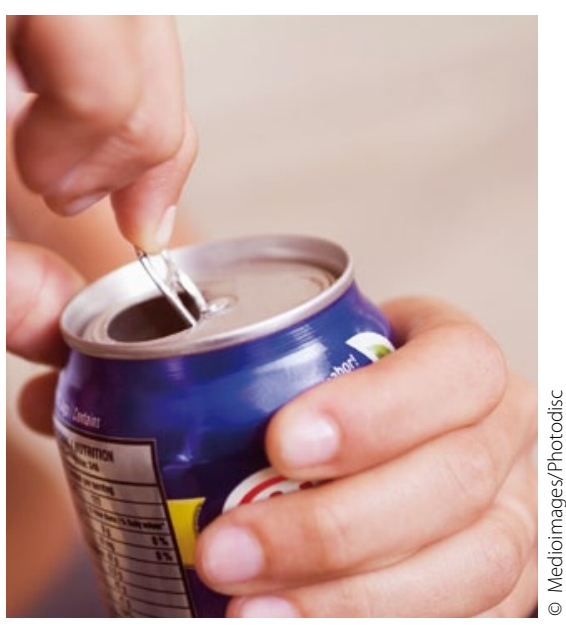

Süße Getränke enthalten viele Kalorien, machen aber nicht satt.

gestellt. Im Verlauf der Studie stieg der z-Score des BMI in der Studiengruppe um 0,02 SI-Einheiten, in der Gruppe mit dem zuckerhaltigen Getränk um 0,15 SI-Einheiten an. Das Körpergewicht hatte im Durchschnitt um 6,35 bzw. 7,37 $\mathrm{kg}$ zugenommen. Auch die Hautfaltendicke, der Quotient aus Taillenumfang und Körpergröße und die Fettmasse hatten in der Gruppe mit dem kalorienfreien Getränk signifikant weniger zugenommen. Bei den 136 Kindern, die die Studie vorzeitig abgebrochen hatten, zeichnete sich ein ähnlicher Trend ab wie bei den 477 Kindern mit regulärer Studienteilnahme.

- J.C. de Ruyter et al.

A trial of sugar-free or sugar-sweetened beverages and body weight in children. New. Engl. J. Med. 2012; 367: 1397-1406

\section{Kommentar}

Die Studie zeigt, dass durch eine einfache Maßnahme, die zudem keinen Verlust an Lebensqualität bedeutet, bereits nach kurzer Zeit Körpergewicht und Fettmasse bei Grundschulkindern signifikant verringert werden kann. Das Hauptproblem der süßen Erfrischungsgetränke dürfte darin liegen, dass sie zwar zusätzliche Kalorien zur Verfügung stellen, jedoch nicht zu einem Sättigungsgefühl führen, das zu einer verringerten Aufnahme fester Nahrung beiträgt.
Insofern schlagen die zusätzlichen $100 \mathrm{kcal}$ voll zu Buche. Ob die Gewichtszunahme allerdings nur auf die zusätzlichen aufgenommenen Kalorien zurückzuführen ist, erscheint fraglich. Aus anderen Studien ist bekannt, dass Kinder, die süße Erfrischungsgetränke zu sich nehmen, auch mehr Fast Food essen und mehr fernsehen. Insofern sind die süßen Dosen nicht nur Kalorienträger, sondern auch Merkmal eines bestimmten ungesunden Lebensstils. 